\section{Distúrbios metabólicos em doenças infecciosas emergentes e negligenciadas}

\author{
Metabolic disorders in emerging and neglected infectious diseases
}

\author{
Clarisse Mourão Melo Ponte 1,2, Maria Helane Costa Gurgel', \\ Glaydson Assunção Ponte'2, Adriana Valéria Assunção Ramos', \\ Renan Magalhães Montenegro Júnior ${ }^{1}$
}

\section{SUMÁRIO}

A substituição gradual e progressiva das doenças infecciosas e parasitárias pelas doenças crônico-degenerativas como causas de morbidade e mortalidade, caracterizando o processo de transição epidemiológica, não tem sido observada em várias populações, em especial em países subdesenvolvidos ou em desenvolvimento, verificando-se, na realidade, uma sobreposição desses perfis (transição incompleta). Além do aumento da prevalência de distúrbios metabólicos, várias doenças infecciosas permanecem endêmicas em diversas regiões, como é o caso da hanseníase, da tuberculose, da leishmaniose, das hepatites virais, entre outras, assim como condições emergentes nas últimas décadas, como a infecção pelo HIV/Aids. Nesse contexto, nos últimos anos tem sido dada uma maior atenção para a ocorrência de distúrbios metabólicos, principalmente a partir da observação de elevada incidência dessas anormalidades associadas à infecção pelo HIV/Aids e à sua terapia com as drogas antirretrovirais. Nesta revisão são abordados aspectos clínico-epidemiológicos dos distúrbios metabólicos reportados em algumas enfermidades infectoparasitárias de relevância mundial e local (no Brasil), assim como possíveis mecanismos e fatores envolvidos nessas associações. Arq Bras Endocrinol Metab. 2010;54(9):785-92

\section{Descritores}

Infecção; distúrbios metabólicos; diabetes melito; doença cardiovascular; tuberculose; HIV; Aids; hepatite; hanseníase; leishmaniose

\section{SUMMARY}

The gradual and progressive replacement of infectious and parasitic by chronic diseases as causes of morbidity and mortality, characterizing the process of epidemiological transition hasn't been observed in various populations, especially in underdeveloped or developing countries characterizing a superposition of these profiles (incomplete transition). Besides the increased prevalence of metabolic disorders, various infectious diseases remain endemic in several regions, such as leprosy, tuberculosis, leishmaniasis, viral hepatitis, among others, as well as emerging diseases in recent decades, as HIV infection/Aids. In this context, more attention has been given to the occurrence of metabolic disturbances in the recent years, mainly from the observation of a high incidence of metabolic disorders associated with HIV infection/Aids, and its therapy with antiretroviral drugs. This review addresses clinical and epidemiological aspects of metabolic disturbances reported in some infectious and parasitic diseases with worldwide and local (Brazil) relevance, as well as possible mechanisms and factors involved in these associations. Arq Bras Endocrinol Metab. 2010;54(9):785-92

\section{Keywords}

Infection; metabolic disorders; diabetes mellitus; cardiovascular disease; tuberculosis; HIV; Aids; hepatitis; leprosy; leishmaniasis
Faculdade de Medicina, Universidade Federal do Ceará (UFC), Fortaleza, CE, Brasil 2 Hospital São José de Doenças Infecciosas, Secretaria de Saúde do Estado do Ceará, CE, Brasil

Correspondência para: Renan Magalhães Montenegro Júnior

Rua Prof. Costa Mendes, 1608, $5^{\circ}$ andar

60416-200 - Fortaleza, CE, Brasil renanjr@ufc.br

Recebido em 10/12/2010 Aceito em 17/12/2010 


\section{INTRODUÇÃO}

A partir da segunda metade do século XIX, especialAmente entre países desenvolvidos, passou a ocorrer uma substituição gradual e progressiva das doenças infecciosas e parasitárias por doenças crônico-degenerativas como causas de morbidade e mortalidade. Não obstante, em várias populações, em especial em países subdesenvolvidos ou em desenvolvimento, uma transição linear desses processos não foi observada, verificando-se, na realidade, uma sobreposição desses perfis (transição incompleta) (1). Além do aumento da prevalência de distúrbios metabólicos, como diabetes melito tipo 2 (DM2), dislipidemia, obesidade, hipertensão arterial sistêmica (HAS) e síndrome metabólica $(\mathrm{SM})$, várias doenças infecciosas e parasitárias permanecem endêmicas em diversas regiões, como é o caso da hanseníase, da tuberculose, das leishmanioses, das hepatites virais, entre outras, assim como condições emergentes nas últimas décadas, como a infecção pelo HIV/Aids (1). Nesse cenário, a coexistência de doenças infecciosas e parasitárias com distúrbios metabólicos fez recrudescer o interesse pelas interações existentes entre esses processos, principalmente com a constatação de uma elevada incidência de complicações, em especial aquelas relacionadas a desfechos cardiovasculares (2). Entretanto, essas associações passaram a ser foco de maior atenção nos últimos anos principalmente após a observação da alta incidência de distúrbios metabólicos associados à infecção pelo HIV/Aids (3). Especula-se que as doenças infecciosas e parasitárias, principalmente aquelas de caráter crônico, possam estar relacionadas a uma maior predisposição a anormalidades metabólicas, tendo em vista que promovem um estado de inflamação clínica ou subclínica persistente (4).

Nesta revisão, são abordados aspectos clínico-epidemiológicos dos distúrbios metabólicos reportados em algumas enfermidades infecciosas e parasitárias de relevância mundial e local (no Brasil), assim como possíveis fatores e mecanismos envolvidos nessa associação.

\section{HIV/AIDS}

A infecção pelo HIV/Aids é uma condição claramente associada a distúrbios metabólicos e maior risco para doenças cardiovasculares (DCVs) $(5,6)$. Dados do $M u l-$ ticenter Aids Cohort Study (MACS) evidenciaram um risco quatro vezes maior para o surgimento de DM2 em homens portadores do HIV quando comparados com homens soronegativos, ajustados para idade e índice de massa corpórea (IMC) (7).

Alterações do metabolismo dos lipídeos são altamente prevalentes nesses pacientes $(5,6)$. Sugere-se que a própria infecção pelo HIV desempenha o papel principal na redução do colesterol, principalmente de lipoproteína de alta densidade (HDL), em pacientes não expostos aos antirretrovirais (ARVs), por meio de mecanismos relacionados à replicação viral (8). A proteína Nef do HIV é capaz de inibir o ATP-Binding Cassette Transporter-AI (ABCA-1), responsável pelo efluxo de colesterol dos macrófagos (transporte reverso) (9). Em adição, a diminuição de HDL pode ser explicada pela perda de peso e piora do estado nutricional, além do aumento do catabolismo dessas partículas encontrado nos estados pró-inflamatórios, em que ocorre não apenas diminuição, mas principalmente alterações na composição e função da partícula de HDL (10).

Em pacientes submetidos à terapia antirretroviral (TARV), observa-se aumento de colesterol total (CT), lipoproteína de baixa densidade (LDL) e triglicerídeos (TGs) com persistência da redução de HDL (5). Especula-se que os inibidores de protease (IPs) tenham como alvo a região catalítica da protease do HIV, que apresenta homologia com as sequências de duas proteínas humanas que regulam o metabolismo dos lipídeos: a região C-terminal da proteína citoplasmática ligante do ácido retinoico tipo-l (CRABP-1), responsável pela ativação do receptor ativado de proliferação do peroxissoma- $\gamma$ (PPAR- $\gamma$ ), e a proteína relacionada ao receptor de LDL (LPR), localizada nos hepatócitos e responsável pela absorção hepática de quilomícrons. Assim, os IPs poderiam acarretar alterações metabólicas e lipodistróficas, em decorrência do aumento da apoptose dos adipócitos e da redução da diferenciação de pré-adipócitos em adipócitos, causada pela inibição da expressão do PPAR- $\gamma$ nessas células, com efeito final na redução do armazenamento de triglicerídeos e o aumento da liberação dos ácidos graxos livres (AGLs) pelo tecido adiposo, contribuindo para o aumento da resistência periférica à insulina e obesidade central (11).

No que se refere à HAS, existem controvérsias acerca do aumento de risco para essa condição entre os pacientes com infecção pelo HIV. Os resultados de alguns estudos são contraditórios, mas a maioria deles sugere que não existe uma associação bem estabelecida entre infecção pelo HIV, uso de TARV e desenvolvimento de HAS (12).

Anormalidades na distribuição da gordura corporal têm sido relatadas em até $50 \%$ dos pacientes ambulato- 
riais infectados pelo HIV, sendo essa proporção maior em pacientes em uso da TARV. No entanto, a prevalência de lipodistrofia associada ao HIV (LAHIV), apresentada sob as formas de lipoatrofia, lipo-hipertrofia ou lipodistrofia mista, depende de vários fatores, como tempo de seguimento, uso de ARVs, critérios para seleção de amostras de pacientes e critérios diagnósticos (13).

Inicialmente, os IPs foram mais associados ao surgimento da lipoatrofia. Contudo, nas últimas décadas, tem sido demonstrado que a patogênese da LAHIV é multifatorial. Atualmente se considera que as drogas mais comumente relacionadas à lipoatrofia são os inibidores da transcriptase reversa análogos dos nucleosídeos (ITRNs), principalmente a estavudina, e em menor extensão a zidovudina (14).

Os ITRNs podem inibir não somente a trancriptase reversa viral, mas também a DNA-polimerase gama mitocondrial, interferindo na função e replicação mitocondrial, com consequente inibição da fosforilação oxidativa das células. Isso resulta em redução da produção de adenosina trifosfato (ATP), dano e morte celular, levando ao surgimento das várias manifestações clínicas e metabólicas, incluindo a lipoatrofia (15). Adicionalmente, um recente estudo mostrou inesperada piora da lipoatrofia em pacientes em uso de um inibidor da transcriptase reversa não análogo dos nucleosídeos (ITRNN), o efavirenz, comumente utilizado como droga de primeira linha nos esquemas ARVs (13). Ademais, os IPs podem interferir com o processo de diferenciação de adipócitos e predispor à redução dos adipócitos subcutâneos e acúmulo de adipócitos viscerais (11). Ao contrário da lipoatrofia, o papel dos ARVs na patogênese da lipo-hipertrofia é menos compreendido e não parece estar diretamente relacionado ao seu surgimento (13).

Com relação ao aumento do risco cardiovascular, um grande estudo observacional prospectivo, Data Collection on Adverse events of Anti-HIV Drugs (D:A:D Study), demonstrou aumento do risco relativo de infarto agudo do miocárdio (IAM) em 16\% por ano de exposição à TARV, especialmente entre aqueles em uso de IPs, como indinavir e lopinavir/ritonavir, e em uso de ITRNs, como abacavir e didanosina (16).

Além dos ARVs, dados recentes sustentam que a hipótese de que a infecção pelo HIV per si promove aterosclerose. Resultados do estudo Strategies for Management of Anti-Retroviral Therapy (SMART Study) demonstraram que em pacientes submetidos à interrupção programada da TARV, apesar da melhora parcial dos parâmetros lipídicos, houve maior mortalidade geral e cardiovascular, assim como elevação de citocinas pró-inflamatórias, como interleucina- 6 (IL-6), e aumento de reagentes de fase aguda, como d-dímero, produtos de degradação do fibrinogênio, plasmina e fator XIII (17).

Por fim, o extenso dano à mucosa intestinal induzido pelo vírus favorece a translocação microbiana para a corrente sanguínea, levando a um estado de endotoxemia, como demonstrado pelo aumento de proteínas ligadoras de lipopolissacarídeos (LPS) bacterianos na circulação de pacientes infectados pelo HIV. O estado de endotoxemia induz ativação de linfócitos T e monócitos e pode estar associado ao aumento do risco para DCVs (18).

O tratamento das anormalidades metabólicas associadas ao HIV objetiva, em última análise, a redução do risco cardiovascular e a melhora da qualidade de vida. Assim, a abordagem terapêutica deve ser individualiza$\mathrm{da}$, dirigida às alterações metabólicas presentes, incluindo medidas não farmacológicas e farmacológicas (19).

A abordagem do DM2 e da intolerância à glicose (IG) é, em linhas gerais, semelhante àquela de pacientes sem infecção pelo HIV (19). A metformina (MTF) promove melhor controle glicêmico e redução de risco cardiovascular no cenário de resistência à insulina e é considerada a terapia farmacológica de escolha. Em pacientes intolerantes à glicose e mesmo nos normoglicêmicos, a MTF tem mostrado um papel benéfico sobre o perfil metabólico e a função endotelial (20). Entretanto, alguns estudos têm apontado que essa droga pode acentuar a perda de gordura subcutânea, tornando a lipoatrofia mais evidente (13).

Para o tratamento da dislipidemia, recomenda-se que sejam adotadas modificações de estilo de vida, incluindo dieta e atividade física, e que sejam objetivados os mesmos alvos lipídicos definidos pelo National Cholesterol Education Program Expert Panel on Detection, Evaluation, and Treatment of High Blood Cholesterol in Adults - Adult Treatment Panel III (NCEP-ATPIII). Os fibratos são as drogas de escolha quando os níveis de triglicerídeos excedem $500 \mathrm{mg} / \mathrm{dl}$. Nos pacientes com hipercolesterolemia deve ser considerado o uso das estatinas (19). Deve-se lembrar, contudo, que a maioria das drogas dessa classe é metabolizada por enzimas do complexo citocromo P450, principalmente pela via CYP3A4, e que os IPs são potentes inibidores dessa via, favorecendo o aumento dos níveis séricos das estatinas. Atenção deve ser dada ao tratamento de idosos com 
drogas metabolizadas via complexo citocromo P450, devido ao maior risco de complicações relacionadas à menor metabolização de fármacos nesses pacientes (21).

Algumas estratégias para o tratamento da lipodistrofia vêm sendo estudadas. A substituição de estavudina por outros ITRNs, como tenofovir, pode resultar em reversão parcial da lipoatrofia de forma clinicamente significativa (22). Entre os agentes sintéticos, o metacrilato tem surgido como opção para o preenchimento facial (23).

Quanto ao tratamento da lipo-hipertrofia, modificações do estilo de vida e o uso de MTF têm demonstrado redução do acúmulo de gordura visceral (13). Cirurgias para excisão do tecido adiposo ou lipossucção das áreas com acúmulo anormal de gordura têm sido realizadas em pacientes com importante "corcova de búfalo"; no entanto, a recorrência do acúmulo pode ocorrer (24). A reposição de testosterona em homens hipogonádicos parece resultar em melhora da lipo-hipertrofia visceral, menor risco para desenvolvimento de DM2 e SM $(25,26)$. No entanto, o papel da administração de testosterona sobre o risco cardiovascular permanece incerto (27).

\section{HEPATITES VIRAIS}

Diversos estudos seccionais e longitudinais têm demonstrado aumento de risco de DM2 e de resistência à insulina em pacientes infectados pelo vírus da hepatite C $(\mathrm{HCV})(28,29)$. Em indivíduos cronicamente infectados pelo vírus $\mathrm{C}$ da hepatite, as prevalências de IG e DM2 situam-se em $40 \%$ e $17 \%$, respectivamente (28).

Já quanto à relação entre a infecção pelo vírus $\mathrm{B}$ da hepatite (HBV) e o metabolismo glicídico, as evidências são menos claras. Entretanto, um estudo recente mostrou associação inversa entre os níveis de DNA do HBV e os níveis de HDL e adiponectina em pacientes com hepatite B crônica (30).

Rouabhia e cols. (29) demonstraram, em estudo seccional com 416 pacientes infectados pelos vírus da hepatite B ou C (126 vs. 290 indivíduos, respectivamente), maior prevalência de DM2 em pacientes infectados pelo último $(39,1 \%)$ em comparação aos do primeiro grupo $(5,0 \%)(\mathrm{p}<0,0001)$. Encontraram também que a infecção pelo HCV (razão de chances - odds-ratio [OR] 4,73, 95\% CI: 1,7-13,2) e aumento das enzimas hepáticas (OR 2,22, 95\% CI: 1,1-4,5) foram associados de forma independente a um maior risco de DM2 (29).
Estudos moleculares têm demonstrado que uma proteína do core do HCV pode diretamente inibir a sinalização da insulina e aumentar a produção de espécies reativas de oxigênio, favorecendo a instalação de resistência à insulina (31). $\mathrm{O}$ excesso de fator de necrose tumoral- $\alpha$ (TNF- $\alpha$ ) em pacientes com hepatite C crônica correlaciona-se com uma rápida progressão da fibrose e uma menor resposta ao tratamento com interferon (32). Além disso, o genótipo do $\mathrm{HCV}$ pode ser de importância na ocorrência dos distúrbios metabólicos. Os genótipos 1 e 4 estão significativamente associados com resistência à insulina com mais frequência do que os genótipos 2 e 3 (37\% vs. 17\%) (33).

Mais raramente, outros fenômenos imunológicos podem estar envolvidos na relação entre resistência à insulina e infecção pelo HCV. Daniel e cols. (34) relataram um caso de desenvolvimento de resistência à insulina tipo $\mathrm{B}$ com presença de anticorpos contra o receptor de insulina durante a terapia com interferon-alfa (34).

A associação entre infecção crônica pelo $\mathrm{HCV}$ e resistência à insulina parece ser bidirecional. Além do papel deletério da infecção viral sobre o metabolismo, sugere-se que na resistência à insulina e DM2 o aumento dos níveis de leptina e TNF- $\alpha$ e a redução dos níveis de adiponectina podem desempenhar algum papel na progressão da doença hepática e aceleram o processo de fibrose, favorecendo a evolução para cirrose e hepatocarcinoma (31).

A presença de anormalidades metabólicas tem mostrado influenciar a resposta ao tratamento, sendo este evidenciado em diversos estudos em que a resistência à insulina e a obesidade reduzem a probabilidade de uma resposta virológica sustentada ao tratamento antiviral. Em consonância com esses achados, um estudo recente realizado com pacientes infectados pelo genótipo 1 do HCV mostrou que aqueles com resultados do índice de Modelo de Avaliação da Homeostase - Resistência à Insulina (HOMA-IR) > 2,0 tiveram uma resposta sustentada duas vezes menor ao tratamento que pacientes com HOMA-IR < 2,0 (32,8\% vs. $60,5 \%$, respectivamente) (35). Ainda nesse contexto, observa-se que a resposta virológica sustentada ao tratamento antiviral promove melhora da resistência à insulina e da função das células $\beta$ das ilhotas pancreáticas (28).

Além do tratamento antiviral específico, a abordagem dos vários componentes da SM, com enfoque na redução da resistência à insulina, poderá contribuir para 
maiores taxas de sucesso no tratamento da hepatite C crônica (28). Assim, é importante citar algumas peculiaridades do tratamento da resistência à insulina e DM2 neste contexto. Aproximadamente metade dos pacientes com insuficiência hepática apresenta algum grau de desnutrição e uma parcela desses pode ter doença hepática avançada, aumentando o risco de episódios de hipoglicemia. Além disso, a maioria dos antidiabéticos orais é metabolizada pelo fígado e, portanto, os níveis de glicose devem ser cuidadosamente monitorados durante o tratamento com essas drogas (36).

A droga de escolha para o tratamento da RI nos pacientes diabéticos com hepatite C é a MTF (28). Um recente estudo controlado, randomizado, duplo-cego, avaliou os efeitos da adição de MTF à terapia padrão para a hepatite C. Esse estudo demonstrou que mulheres com infecção causada pelo genótipo 1 com HOMA-IR > 2,0 tratadas com MTF apresentaram uma maior redução da carga viral durante as primeiras 12 semanas em comparação ao grupo que não recebeu MTF (37). No entanto, ressalta-se que a MTF é relativamente contraindicada em pacientes com insuficiência hepática avançada e nos pacientes que continuam a ingerir álcool, em função do risco aumentado de acidose lática (28).

Apesar de serem relativamente seguras em pacientes com hepatopatias, as sulfonilureias têm utilidade mais limitada, pois não atuam sobre a sensibilidade à insulina (28). Os inibidores de alfa-glicosidase, como a acarbose, podem ser úteis em pacientes com insuficiência hepática, uma vez que reduzem a absorção de carboidratos no intestino. Em um estudo randomizado, controlado com placebo, envolvendo pacientes com encefalopatia hepática, a acarbose produziu uma melhora significativa nos níveis pós-prandiais de glicose. Além disso, os pacientes tiveram uma redução dos níveis plasmáticos de amônia e aumento da frequência de evacuações, condições associadas à redução do risco de encefalopatia hepática (38).

\section{ENTEROVIROSES}

Fatores ambientais, como toxinas, antígenos alimentares e, sobretudo, infecções virais, têm sido implicados na etiopatogênese do diabetes melito l (DMI). Estudos experimentais e clínicos apontam que diversas viroses, como enteroviroses, rubéola, caxumba, infecção por rotavírus, parvovirose e citomegalovirose, podem desempenhar algum papel na patogênese do DMl (39).

Neste contexto, nos últimos anos, a maioria dos estudos tem-se centrado nas enteroviroses. Os enterovírus humanos compreendem um grande grupo de agentes patogênicos que se subdividem em quatro espécies A, B, C e D, com mais de 100 sorotipos diferentes. Todos os sorotipos investigados até o momento parecem incluir cepas com a capacidade de danificar as células $\beta$ (40). Os enterovírus apresentam tropismo pela ilhota pancreática, como demonstrado pela detecção do RNA viral por hibridização in situ e pela identificação de proteínas virais em amostras de pâncreas de pacientes com DMl (4l).

Apesar de os estudos epidemiológicos apontarem uma associação entre enteroviroses e DMl e de os estudos em animais e humanos demonstrarem a presença de citólise nas ilhotas pancreáticas em decorrência do processo viral, uma correlação direta entre esse achado e o desenvolvimento de DMl ainda não está clara (42).

\section{TUBERCULOSE}

A associação entre diabetes e tuberculose é reconhecida há vários anos. Estudos têm demonstrado um risco aumentado dessa infecção tanto em indivíduos com DMl quanto naqueles com DM2. Stevenson e cols., em uma revisão baseada em sistemática, observaram que o diabetes implicou um RR ou uma razão de chance para tuberculose de 1,5 a 7,8 vezes (43). Em outra recente revisão, baseada em metanálise de 13 estudos observacionais, a presença de diabetes foi associada a um risco relativo (RR) de 3,11 para a infecção ativa por tuberculose (44).

Embora a maioria dos estudos aponte o DM como um fator de risco para a tuberculose, essa relação é possivelmente bidirecional (45). Evidências demonstraram aumento do risco para desenvolvimento de diabetes em indivíduos com tuberculose (45). Especula-se que a resposta imune modulada por interleucinas e TNF- $\alpha$ pode promover uma redução da sensibilidade e da secreção de insulina, o que justificaria o surgimento de IG ou DM2 nos pacientes infectados (43). Não obstante, ainda faltam estudos longitudinais para melhor definição dessa associação. Por outro lado, é bem estabelecido que a tuberculose, assim como algumas das suas terapêuticas, possui efeito hiperglicemiante e dificulta o controle metabólico do paciente com diabetes (46).

A terapia tuberculostática em pacientes diabéticos merece atenção especial, uma vez que esses pacientes 
apresentam elevada prevalência de comorbidades, o que aumenta o risco de toxicidades e interações medicamentosas. Ademais, há a necessidade de maior atenção no manejo farmacológico dos pacientes com coinfecção pelo HIV/Aids, comumente polimedicados, conforme já abordado em seção anterior (47).

O DM pode alterar a farmacocinética das drogas utilizadas para o tratamento da tuberculose (48). Em um estudo realizado em pacientes com tuberculose foi demonstrado que os diabéticos apresentavam níveis séricos de rifampicina $53 \%$ menores quando comparados aos não diabéticos, alertando para o maior risco de falha terapêutica e desenvolvimento de resistência aos fármacos antituberculose (49).

Por outro lado, o tratamento com rifampicina pode causar hiperglicemia por meio de interações com os hipoglicemiantes orais. Esse fármaco é um potente indutor de uma série de enzimas do sistema do citocromo P450 (50). A indução dessas enzimas pode levar a aumento da metabolização das drogas administradas em concomitância com a rifampicina, reduzindo seus efeitos. As sulfonilureias, como, por exemplo, glibenclamida e glipizida, que são substratos das vias enzimáticas do citocromo P4502C9 (CYP2C9), têm suas concentrações séricas reduzidas em $39 \%$ e $22 \%$, respectivamente, quando coadministradas com rifampicina (5l).

As necessidades de insulina podem aumentar em indivíduos em uso de rifampicina, uma vez que em uma fase inicial do tratamento com esse fármaco pode ocorrer um estado de hiperglicemia associado à hiperinsulinemia, mesmo em indivíduos não diabéticos (52). Portanto, pacientes em uso de rifampicina devem realizar rigoroso monitoramento glicêmico para ajuste adequado dos agentes antidiabéticos (45).

Em pacientes diabéticos em uso de isoniazida, fármaco de primeira linha no tratamento da tuberculose, recomenda-se o uso associado de piridoxina em razão do maior risco de neuropatia periférica (47).

\section{HANSENÍASE}

Estudos acerca da relação entre hanseníase e alterações metabólicas são escassos. No entanto, alguns autores têm demonstrado maior prevalência de DM2 em pacientes com hanseníase. Em estudo realizado em serviço de referência em dermatologia, encontrou-se prevalência de DM2 de 14,2\% em portadores de hanseníase versus 2,0\% no grupo controle. Nesta série, a maior frequência de diabetes $(19,3 \%)$ foi verificada em pacientes com a forma virchowiana, ao passo que uma menor proporção foi observada entre os pacientes com a forma tuberculoide $(6,4 \%)$. Observou-se ainda uma diminuição dos níveis glicêmicos após o tratamento da hanseníase, sugerindo uma possível associação entre a infecção pela Mycobacterium leprae e o metabolismo dos carboidratos (53). Em adição, os fármacos utilizados para o tratamento da hanseníase e dos estados reacionais, como corticosteroides e a rifampicina, podem ter ações deletérias sobre o controle glicêmico de portadores de DM2 (47).

\section{HELMINTOSES INTESTINAIS}

Sabe-se que as helmintoses são um problema de saúde mundial. Além de causarem diretamente infecção intestinal, esses parasitas afetam a incidência e a progressão de outras doenças, mediante os efeitos imunomodulatórios. A partir de estudos em modelos animais, tem sido sugerida uma relação inversa entre infecção por helmintos e doenças autoimunes, inflamatórias e alérgicas (54).

Há vários anos tem sido investigada a relação entre infecção por Schistosoma mansoni e diabetes (55). Recentemente, alguns autores têm mostrado um papel protetor da esquistossomose sobre o risco de desenvolvimento de DMl. Cooke e cols. (56), em estudo experimental com modelos animais geneticamente predispostos ao desenvolvimento de DM autoimune (camundongos diabéticos não obesos), demonstraram que uma alteração da resposta imune Thl por Th2 observada em camundongos infectados por Schistosoma mansoni está associada com uma redução da incidência de DMl, afecção reconhecidamente mediada por resposta Thl contra os antígenos das células $\beta$ das ilhotas pancreáticas. Especula-se que o predomínio de uma resposta Th2 determinaria diferentes respostas imunológicas aos agentes ou antígenos, modulando a expressão das doenças autoimunes. Dessa forma, sugerem que o aumento da incidência de doenças autoimunes em populações geneticamente predispostas, como, por exemplo, ao DMl, possivelmente estaria relacionado à menor prevalência de doenças infectoparasitárias observada em alguns países, nas últimas décadas (56).

A esquistossomose tem sido também relacionada com alterações do metabolismo lipídico. Lima e cols. (57) publicaram estudo demonstrando redução da atividade plasmática da lecitina: colesterol acetiltransferase (LCAT) em animais infectados por Schistosoma mansoni, com consequente redução dos ésteres de colesterol, aumento 
de fosfolipídeos plasmáticos e do colesterol eritrocitário. As implicações clínicas desses achados são incertas (57).

\section{LEISHMANIOSE}

Há escassos dados publicados sobre relações de distúrbios metabólicos e leishmaniose. A maioria desses são relatos relacionando o desenvolvimento de $\mathrm{DM}$ ao tratamento da leishmaniose com o isotionato de pentamidina, uma droga potencialmente tóxica para as ilhotas pancreáticas (58).

Distúrbios no metabolismo lipídico também têm sido reportados em casos isolados de pacientes acometidos por essa infecção. Há quase duas décadas, Bertoli e cols. descreveram a presença de hipertrigliceridemia em pacientes com leishmaniose visceral (59). Mais recentemente, Liberopoulos e cols. publicaram um relato de um paciente com diagnóstico de leishmaniose visceral que apresentava hipertrigliceridemia e hipocolesterolemia grave com redução de HDL, LDL, apolipoproteínas AI e B e lipoproteína (a) (60). Contudo, faltam estudos que possam estabelecer uma associação entre essas enfermidades.

\section{CONSIDERAÇÕES FINAIS}

A ocorrência (além de fatores e mecanismos possivelmente envolvidos) de distúrbios metabólicos em algumas doenças infecciosas emergentes, como na infecção pelo HIV/Aids, está bem determinada, situação essa que aponta para a necessidade do desenvolvimento de estratégias que favoreçam a redução de tais complicações em pacientes acometidos, especialmente pelas suas potenciais repercussões cardiovasculares. No entanto, em outras condições menos prevalentes, ou mesmo endêmicas, porém negligenciadas (em países subdesenvolvidos e em desenvolvimento), há ainda escassez de dados quanto a essas associações, principalmente de estudos prospectivos controlados. Ações nesse sentido revelam-se importantes a fim de que se tenham dados que subsidiem medidas para melhor abordagem dos pacientes portadores de tais enfermidades.

Declaração: os autores declaram não haver conflitos de interesse científico neste estudo.

\section{REFERÊNCIAS}

1. Pontes RJ, Ramos-Júnior AN, Kerr LRS, Bosi MLM. Transição demográfica e epidemiológica. In: Medronho RA, Bloch KV, Luiz RR,
Werneck GL. Epidemiologia. 2.ed. São Paulo: Atheneu; 2009. p. 123-52.

2. Maia IL, Nicolau JC, Machado MN, Maia LN, Takakura IT, Rocha PRF, et al. Prevalência de Chlamydia Pneumoniae e Mycoplasma Pneumoniae em diferentes formas da doença coronariana. Arq Bras Cardiol. 2009;92(6):439-45.

3. Hadigan C, Meigs JB, Corcoran C, Rietschel P, Piecuch S, Basgoz $\mathrm{N}$, et al. Metabolic abnormalities and cardiovascular disease risk factors in adults with human immunodeficiency virus infection and lipodystrophy. Clin Infect Dis. 2001;32(1):130-9.

4. Hotamisligil GS. Inflammation and metabolic disorders. Nature. 2006;444(7121):860-7.

5. Domingos H, Cunha RV, Paniago AMM, Martins DM, Elkhoury EB, Souza AS. Metabolic effects associated to the highly active antiretroviral therapy (HAART) in AIDS patients. Braz J Infect Dis. 2009;13(2):130-6.

6. Kramer AS, Lazzarotto AR, Sprinz E, Manfroi WC. Alterações metabólicas, terapia antirretroviral e doença cardiovascular em idosos portadores de HIV. Arq Bras Cardiol. 2009;93(5):561-8.

7. Brown TT, Cole SR, Li X, Kingsley LA, Palella FJ, Riddler SA, et al. Antiretroviral therapy and the prevalence and incidence of diabetes mellitus in the multicenter Aids cohort study. Arch Intern Med. 2005;165(10):1179-84.

8. Rose H, Woolley I, Hoy J, Dart A, Bryant B, Mijch A, Sviridov D. HIV infection and high-density lipoprotein: the effect of disease vs the effect of treatment. Metabolism. 2006;55(1):90-95.

9. Hsue PY, Hunt PW, Schnell A, Kalapus SC, Hoh R, Ganz P, et al. Role of viral replication, antiretroviral therapy, and immunodeficiency in HIV-associated atherosclerosis. AIDS. 2009;23(9):1059-67.

10. Oh J, Hegele RA. HIV-associated dyslipidaemia: pathogenesis and treatment. Lancet Infect Dis. 2007;7(12):787-96.

11. Leow MK, Addy CL, Mantzoros CS. Human immunodeficiency virus/highly active antiretroviral therapy-associated metabolic syndrome: clinical presentation, pathophysiology, and therapeutic strategies. J Clin Endocrinol Metab. 2003;88(5):1961-76.

12. Grandominico CJ, Fichtenbaum JM. Short-term effect of HAART on blood pressure in HIV-infected individuals. HIV Clin Trials. 2008;9(1):52-60.

13. Brown TT. Approach to the human immunodeficiency virus-infected patient with lipodystrophy. J Clin Endocrinol Metab. 2008;93(8):2937-45.

14. Grinspoon S, Carr A. Cardiovascular risk and body-fat abnormalities in HIV-infected adults. N Engl J Med. 2005;352(1):48-62.

15. Kotler DP, Rosenbaum K, Wang J, Pierson RN. Studies of body composition and fat distribution in HIV-infected and control subjects. J Acquir Immune Defic Syndr Hum Retrovirol. 1999;20(3):228-37.

16. DAD Study Group, Friis-Møller N, Reiss P, Sabin CA, Weber R, Monforte $A$, et al. Class of antiretroviral drugs and the risk of myocardial infarction. N Engl J Med. 2007;356(17):1723-35.

17. Tebas $P$, Henry WK, Matining R, Weng-Cherng D, Schmitz J, Valdez $H$, et al. Metabolic and immune activation effects of treatment interruption in chronic HIV-1 infection: implications for cardiovascular risk. PLoS One. 2008;3(4):e2021.

18. Crowe SM, Westhorpe CL, Mukhamedova N, Jaworowski A, Sviridov $D$, Bukrinsky $M$. The macrophage: the interection between HIV infection and atherosclerosis. J Leuk Biol. 2010;87(1):589-98.

19. Hoffman RM, Currier JS. Management of antiretroviral treatment-related complications. Infect Dis Clin N Am. 2007;21(1):103-32.

20. Lima LMA, Wiernsperger N, Kraemer-Aguiar LG, Bouskela E. Short-term treatment with metformin improves the cardiovascular risk profile in first-degree relatives of subjects with type 2 diabetes mellitus who have a metabolic syndrome and normal glucose tolerance without changes in c-reactive protein or fibrinogen. Clinics. 2009;64(5):415-20. 
21. Cabrera MAS, Dip RM, Furlan MO, Rodrigues SL. Use of drugs that act on the cytochrome p450 system in the elderly. Clinics. 2009;64(4):273-8.

22. Moyle GJ, Sabin CA, Cartledge J, Johnson M, Wilkins E, Churchill $D$, et al. A randomized comparative trial of tenofovir DF or abacavir as replacement for a thymidine analogue in persons with lipoatrophy. Aids. 2006;20(16):2043-50.

23. Guaraldi G, Orlando G, De Fazio D, De Lorenzi I, Rottino A, De Santis G, et al. Comparison of three different interventions for the correction of HIV-associated facial lipoatrophy: a prospective study. AntivirTher. 2005;10(6):753-9.

24. Behrens GM. Cardiovascular risk and body-fat abnormalities in HIV-infected adults. N Engl J Med. 2005;352(16):1721-2.

25. Saad F. The role of testosterone in type 2 diabetes and metabolic syndrome in men. Arq Bras Endocrinol Metab. 2009;53(8):901-7.

26. Ponte CMM, Gurgel MHC, Montenegro Jr. RM. Gonadotrophic axis dysfunction in men with HIV-infection/Aids. Arq Bras Endocrinol Metab. 2009;53(8):983-8.

27. Sá EQC, Sá FCF, Guedes AD, Verreschi ITN. Testosterona sérica e doença cardiovascular em homens. Arq Bras Endocrinol Metab. 2009;53(8):915-22

28. Garcia-Compean D, Jaquez-Quintana JO, Gonzalez-Gonzalez JA, Maldonado-Garza H. Liver cirrhosis and diabetes: risk factors, phatophysiology, clinical implications and management. World J Gastroenterol. 2009:15(3):280-8.

29. Rouabhia S, Malek R, Bounecer H, Dekaken A, Bendali Amor F, Sadelaoud M, et al. Prevalence of type 2 diabetes in Algerian patients with hepatitis C virus infection. World J Gastroenterol. 2010;16(27):3427-31.

30. Mohamadkhani A, Sayemiri K, Ghanbari R, Elahi E, Poustchi H, Montazeri G. The inverse association of serum HBV DNA level with HDL and adiponectin in chronic hepatitis B infection. Virol J. 2010;14(7):228.

31. Serfaty L, Capeau J. Liver Int. Hepatitis C, insulin resistance and diabetes: clinical and pathogenic data. Liver Int. 2009;29(Suppl2):13-25.

32. Lecube A, Hernandez C, Genesca J, Simo R. Proinflammatory, cytokines, insulin resistance, and insulin secretion in chronic hepatitis C patients: a case-control study. Diabetes Care. 2006;29(5):1096-101.

33. Moucari R, Asselah T, Cazals-Hatem D, Voitot H, Boyer N, Ripault $\mathrm{MP}$, et al. Insulin resistance in chronic hepatitis $\mathrm{C}$ : association with genotypes 1 and 4, serum HCV RNA level, and liver fibrosis. Gastroenterology. 2008;134(2):416-23.

34. Daniel AL, Houlihan JL, Blum JS, Walsh JP. Type B insulin resistance developing during interferon-alpha therapy. Endocr Pract. 2009;15(2):153-7.

35. Romero-Gomez M, Del Mar Viloria M, Andrade RJ, Salmeron J, Diago M, Fernandez-Rodriguez CM, et al. Insulin resistance impairs sustained response rate to peginterferon plus ribavirin in chronic hepatitis C patients. Gastroenterology. 2005;128(3):636-41.

36. Tolman KG, Fonseca V, Dalpiaz A, Tan MH. Spectrum of liver disease in type 2 diabetes and management of patients with diabetes and liver disease. Diabetes Care. 2007;30(3):734-43.

37. Del Campo JA, López RA, Romero-Gómez M. Insulin resistance and response to antiviral therapy in chronic hepatitis $\mathrm{C}$ : mechanisms and management. Dig Dis. 2010;28(1):285-93.

38. Gentile S, Guarino G, Romano M, Alagia IA, Fierro M, Annunziata $S$, et al. A randomized controlled trial of acarbose in hepatic encephalopathy. Clin Gastroenterol Hepatol. 2005;3(2):184-91.

39. Roivainen $\mathrm{M}$, Klingel K. Virus infections and type 1 diabetes risk. Curr Diab Rep. 2010;10(5):350-6.

40. Hober D, Sane F. Enteroviral pathogenesis of type 1 diabetes. Discov Med. 2010;10(51):151-60.
41. Ylipaasto $\mathrm{P}$, Klingel K, Lindberg AM, Otonkoski T, Kandolf R, Hovi $\mathrm{T}$, et al. Enterovirus infection in human pancreatic islet cells, islet tropism in vivo and receptor involvement in cultured islet beta cells. Diabetologia. 2004;47(2):225-39.

42. Goldberg E, Krause I. Infection and type 1 diabetes mellitus - a two edged sword? Autoimmun Rev. 2009;8(8):682-6.

43. Stevenson CR, Critchley JA, Forouhi NG, Roglic G, Williams BG, Dye $C$, et al. Diabetes and the risk of tuberculosis: a neglected threat to public health? Chronic IIIn. 2007;3(3):228-45.

44. Jeon C, Murray M. Diabetes mellitus increases the risk of active tuberculosis: a systematic review of 13 observational studies. PLoS Med. 2008;5(7):e152.

45. Pickup JC. Inflammation and activated innate immunity in the pathogenesis of type 2 diabetes. Diabetes Care. 2004;27(3):813-23.

46. Dooley K, Chaisson R. Tuberculosis and diabetes mellitus: convergence of two epidemics. Lancet Infect Dis. 2009;9(12):737-46.

47. Miranda AE, Golub JE, Lucena FF, Maciel EN, Gurgel MF, Dietze R. Tuberculosis and AIDS co-morbidity in Brazil: linkage of the tuberculosis and AIDS databases. Braz J Infect Dis. 2009;13(2):137-41.

48. Gwilt PR, Nahhas RR, Tracewell WG. The effects of diabetes mellitus on pharmacokinetics and pharmacodynamics in humans. Clin Pharmacokinet. 1991;20(6):477-90.

49. Nijland HM, Ruslami R, Stalenhoef JE, Nelwan EJ, Alisjahbana $\mathrm{B}$, Nelwan $\mathrm{RH}$, et al. Exposure to rifampicin is strongly reduced in patients with tuberculosis and type 2 diabetes. Clin Infect Dis. 2006;43(7):848-54.

50. Niemi M, Backman JT, Fromm MF, Neuvonen PJ, Kivisto KT. Pharmacokinetic interactions with rifampicin: clinical relevance. Clin Pharmacokinet. 2003;42(9):819-50.

51. Niemi M, Backman JT, Neuvonen M, Neuvonen PJ, Kivisto KT. Effects of rifampin on the pharmacokinetics and pharmacodynamics of glyburide and glipizide. Clin Pharmacol Ther. 2001;69(6):400-6.

52. Atkin S, Masson E, Bodmer C, Walker B, White M. Increased insulin requirement in a patient with type 1 diabetes on rifampicin. Diab Med. 1992;10(4):202.

53. Dalpino D, Magna LA, Opromolla DV. Avaliação dos níveis de lipídeos, lipoproteínas, apoproteínas e o papel da lipoproteína (a) no desenvolvimento de aterosclerose e alterações fibrinolíticas nos pacientes portadores de hanseníase virchowiana. Hansen Int. 1997;22(2):20-30.

54. Chatenoud L, You S, Okada H, Kuhn C, Michaud B, Bach JF. 99th Dahlem conference on infection, inflammation and chronic inflammatory disorders: immune therapies of type 1 diabetes: new opportunities based on the hygiene hypothesis. Clin Exp Immunol. 2010;160(1):106-12.

55. Bibawi E. Schistosomiasis and diabetes mellitus. Trans R SocTrop Med Hyg. 1970;64(6):907-8.

56. Cooke A, Tonks $\mathrm{P}$, Jones FM, O'Shea $\mathrm{H}$, Hutchings $\mathrm{P}$, Fulford AJ, et al. Infection with Schistosoma mansoni prevents insulin dependent diabetes mellitus in non-obese diabetic mice. Parasite Immunol. 1999;21(4):169-76.

57. Lima VL, Sena VL, Stewart B, Owen JS, Dolphin PJ. An evaluation of the marmoset Callithrix jacchus (sagüi) as an experimental model for the dyslipoproteinemia of human Schistosomiasis mansoni. Biochim Biophys Acta. 1998;1393(2-3):235-43.

58. Belehu A, Naafs B. Diabetes mellitus associated with pentamidine mesylate. Lancet. 1982;1(8287):1463-4.

59. Bertoli A, Greco AV, Caputo S, Caradonna P, Grieco A, Laghi V. Visceral leishmaniasis presenting with hypertrigliceridaemia. Lancet. 1982;2(8296):504-5.

60. Liberopoulos E, Alexandridis G, Bairaktari E, Elisaf M. Severe hypocholesterolemia with reduced serum lipoprotein(a) in a patient with visceral leishmaniasis. Ann Clin Lab Sci. 2002;32(3):305-8. 